\title{
Halbach Magnetic Circuit for Voice Coil Motor in Hard Disk Drives
}

\author{
Young-Man Choi ${ }^{1}$, Dahoon Ahn ${ }^{1}$, Dae-Gab Gweon ${ }^{1}$, and Jaehwa Jeong ${ }^{2 *}$ \\ ${ }^{1}$ Department of Mechanical Engineering, KAIST, Daejeon 305-701, Korea \\ ${ }^{2}$ Department of Control and Instrumentation Engineering, Korea University, Chungnam 339-700, Korea
}

(Received 17 May 2010, Received in final form 24 July 2010, Accepted 26 July 2010)

\begin{abstract}
Rotary-type voice coil motors are widely used as actuators in hard disk drives. The recent trend toward higher density and smaller form factors in data storage devices requires performance improvement of the voice coil motor. In this study, we introduce a Halbach magnet array to the voice coil motor in order to increase the force generation. The Halbach magnetic circuit outperforms the conventional magnetic circuit due to the confined magnetic flux. To investigate the performance of the Halbach magnetic circuit, we analyze air gap flux density with the various shapes and thickness of the magnets using 3-dimensional finite element analysis. Consequently the optimum shape of the Halbach magnetic circuit is proposed. Simulations and experimental results proved effectiveness of the proposed magnet array in the voice coil motor for a commercial hard disk drive.
\end{abstract}

Keywords : Halbach magnet array, voice coil motor, hard disk drive

\section{Introduction}

A voice coil motor (VCM) is a simple electromagnetic actuator composed of coils, permanent magnets, and iron yokes. The VCM offers excellent control characteristics where direct-drive linear actuation is required. Sometimes, a rotary-type VCM can be utilized as a high performance laser shutter [1]. The most common application of a rotary-type VCM is a hard disk drive (HDD) [2]. The VCM plays an important role of moving read/write heads for seeking and following the destination track in the presence of several disturbances. As demand increases for smaller HDD with larger capacities, a high performance actuator needs to be developed. Lee et al. [3] redesigned the coil shape to enhance dynamic performance. Suzuki et al. [4] proposed a round coil and axe-shaped magnet to reduce out-of-plane force causing a vibration mode. More fundamentally, to make the system move faster, larger actuating force is required with limited volume.

Since VCM utilizes Lorentz force, high magnetic flux density in the air gap generates large actuating force and eventually decreases seek time. In this study, we introduced the Halbach magnet array to increase the air gap flux density of a HDD VCM. In order to evaluate the

*Corresponding author: Tel: +82-41-860-1765

Fax: +82-41-865-1820, e-mail: jaehwa@korea.ac.kr effectiveness of the Halbach magnetic circuit practically, a magnetic circuit from a commercial HDD was replaced with the proposed Halbach magnetic circuit except coil. Then, we provided quantitative evaluation of the performance improvement on a commercial HDD VCM by measuring air gap flux density and dynamic performance. This paper is organized as follows. In section 2, Halbach magnetic circuit is introduced, also together with conventional magnetic circuit. Section 3 describes analysis on various Halbach magnetic circuits. Experimental verification is presented in Section 4. Section 5 concludes this paper.

\section{Halbach Magnetic Circuit}

Since its invention by Halbach [5], many researchers have applied the Halbach magnet array in an electric motor [6], a nuclear magnetic resonance (NMR) [7], and an optical disk drive [8]. In our previous work, we proved the effectiveness of the Halbach magnet array as a magnetic circuit for a rotary-type VCM in terms of generated force and compactness [9]. Also we proposed a Halbach magnetic circuit and analyzed its performance in a labmade VCM [10]. Fig. 1 illustrates the structures of conventional magnetic circuit and Halbach magnetic circuit for a rotary-type VCM. The arrows on the magnets in Fig. $1(\mathrm{c})$ and (d) indicate magnetization directions of each 


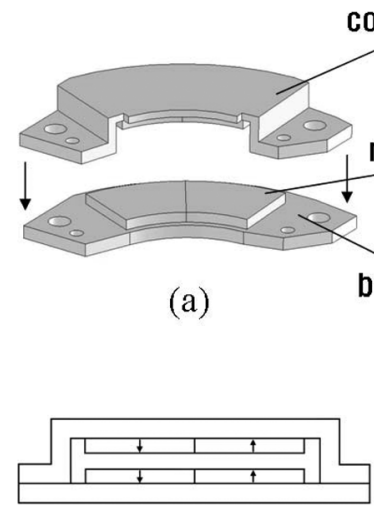

(c)

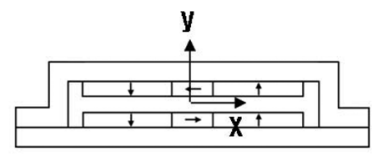

(d)
Fig. 1. Magnetic circuits in rotary-type VCM: (a) Conventional magnetic circuit, (b) Halbach magnetic circuit, (c) cross-sectional view of conventional magnetic circuit, (d) cross-sectional view of Halbach magnetic circuit.

magnet. The conventional magnetic circuit uses vertically-magnetized magnets only. However, the Halbach magnetic circuit includes a horizontally-magnetized magnet between vertically-magnetized magnets. The horizontallymagnetized magnet provides additional magnetic flux paths, and the flux can be less concentrated in the yoke on which the magnets are attached. This allows us to decrease the thickness of the yoke that doesn't make magnetic flux saturated, and gives a possibility to increase the thickness of magnets without changing the total thickness of the magnetic circuit. Consequently, the flux density in the air gap can be increased. We also note that the total area of the magnets and the air gap thickness of the coils do not change.

\section{Analysis}

To evaluate the Halbach magnet array on a rotary-type VCM, we first compared both magnetic circuits using the three-dimensional finite element analysis (3D FEA). The magnetic analysis was performed using a FEA model, which is shown in Fig. 2, implemented by a nonlinear 3D FEA software (MAXWELL 3D, ANSOFT). All magnets, yokes and air gap were meshed by tetrahedral elements. About 48,200 elements were generated for this FEA model. Simulated permanent magnets were NdFeB50-M grade magnets with an intrinsic coercive force of $875 \mathrm{kA} /$ $\mathrm{m}$ and remnant magnetization of $1.39 \mathrm{~T}$. The yoke was assumed as steel 1010. We analyzed Halbach magnetic circuits for the cases of $\theta=0^{\circ}, 8^{\circ}, 12^{\circ}, 16^{\circ}, 20^{\circ}$, and $24^{\circ}$ where $\theta$ is the opening angle of the fan-shaped horizontally magnetized magnet as shown in Fig. 1(b). The Halbach magnetic circuit with $\theta=0^{\circ}$ represents a conv-

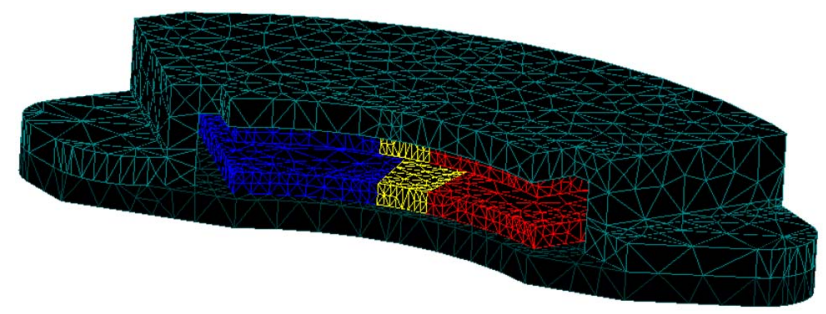

Fig. 2. FEA model of double-sided Halbach magnet array.

Table 1. Magnetic flux densities for various opening angles.

\begin{tabular}{ccc}
\hline \hline $\begin{array}{c}\text { Opening angle } \\
\text { of center magnet }\end{array}$ & Air gap (T) & Yoke (T) \\
\hline $0^{\circ}$ & 0.7280 & 1.649 \\
$8^{\circ}$ & 0.7299 & 1.422 \\
$12^{\circ}$ & 0.7302 & 1.343 \\
$16^{\circ}$ & 0.7310 & 1.254 \\
$20^{\circ}$ & 0.7313 & 1.143 \\
$24^{\circ}$ & 0.7313 & 1.016 \\
\hline
\end{tabular}

entional magnetic circuit. From the FEA results, the size of the horizontally-magnetized magnet was determined. Fig. 3 shows the simulated y-directional component of the flux density in the middle of the air gap along the $\mathrm{X}$-axis in the cross-sectional view of the Halbach magnetic circuit shown in Fig. 1(d). Table 1 lists the flux densities in air gap and yoke for various opening angles. Even though the flux density in air gap does not change much, the flux density in yoke is drastically reduced when increasing the opening angle. This phenomenon is very meaningful because we can decrease the thickness of the yoke, and increase the thickness of the magnets without changing the total thickness of the magnetic circuit.

However, a restriction exists. Fig. 3 shows air gap flux

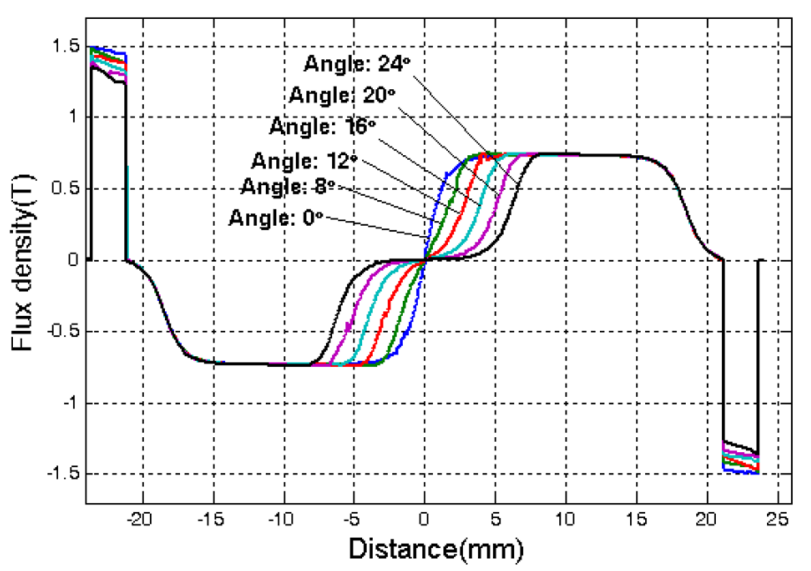

Fig. 3. Simulated air gap flux density of Halbach magnetic circuits with regard to the opening angle of center magnet. 


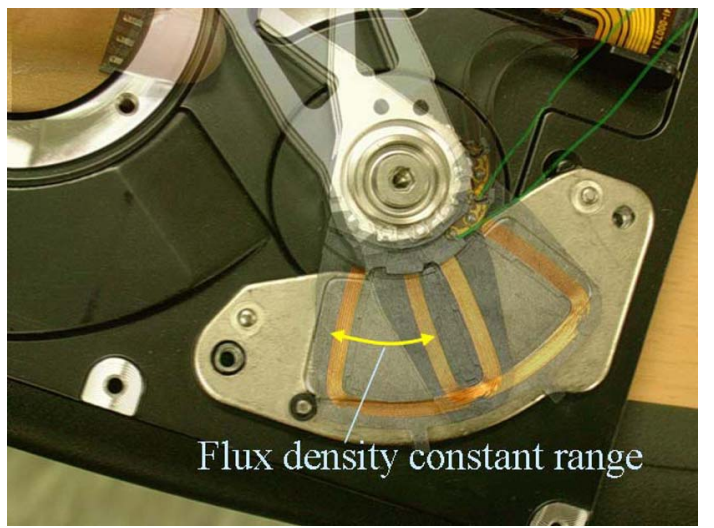

Fig. 4. Picture of voice coil motor showing the flux density constant range.

density along the arc trajectory of the VCM coil. As the angle of the center magnet increases, the flux density constant range - the range over which the coils can move without any flux density variations - decreases. Considering the flux density constant range, the size of the center magnet was determined. Fig. 4 shows a rotary-type VCM and the flux density constant range. The camera was fixed over the HDD, and two pictures were taken when the coil was at the both ends of the working range of the VCM. Overlapping two pictures, the flux density constant range was determined. From Fig. 3, we found that the length of the flux density constant range should be $12.5 \mathrm{~mm}$ at least. To satisfy this condition, the size of the center

Table 2. Analysis results of conventional magnetic circuit and Halbach magnetic circuit.

\begin{tabular}{ccccc}
\hline \hline & $\begin{array}{c}\text { Thickness } \\
\text { of magnets } \\
(\mathrm{mm})\end{array}$ & $\begin{array}{c}\text { Thickness } \\
\text { of yokes } \\
(\mathrm{mm})\end{array}$ & $\begin{array}{c}\text { Flux density } \\
\text { in the air gap } \\
(\mathrm{T})\end{array}$ & $\begin{array}{c}\text { Flux density } \\
\text { in the base } \\
\text { yoke }(\mathrm{T})\end{array}$ \\
\hline $\begin{array}{c}\text { Conventional } \\
\text { magnetic } \\
\text { circuit }\left(\theta=0^{\circ}\right)\end{array}$ & 1.8 & 2.4 & 0.728 & 1.649 \\
\hline \multirow{6}{*}{ Halbach } & 1.8 & 2.4 & 0.730 & 1.343 \\
magnetic & 2.9 & 2.3 & 0.748 & 1.380 \\
circuit & 2.3 & 1.9 & 0.809 & 1.413 \\
$\left(\theta=12^{\circ}\right)$ & 2.4 & $\mathbf{1 . 8}$ & $\mathbf{0 . 8 1 0}$ & $\mathbf{1 . 6 6 1}$ \\
& 2.5 & 1.7 & 0.804 & 1.740 \\
& 2.6 & 1.6 & 0.778 & 1.747 \\
& 2.7 & 1.5 & 0.773 & 1.761 \\
& 2.8 & 1.4 & 0.765 & 1.769 \\
& 2.9 & 1.3 & 0.752 & 1.773 \\
& 3.0 & 1.2 & 0.741 & 1.773 \\
\hline
\end{tabular}

magnet, $\theta$, was chosen as $12^{\circ}$.

From the previous result, we knew that the Halbach magnet array reduced flux density in yoke, which gives a capacity to use thicker magnet. Thus, various sets of magnet and yoke were simulated keeping the total thickness constant. Table 2 lists the results including the conventional case. With the conventional magnetic circuit, the thicknesses of the magnets and yokes were $1.8 \mathrm{~mm}$ and $2.4 \mathrm{~mm}$, respectively. In this case, the air gap flux density was $0.728 \mathrm{~T}$ and the flux density in the base yoke was 1.649 T. With the Halbach magnetic circuit, the air gap flux density has an optimal value for a certain set. As the thickness of magnets is increased, the air gap flux density becomes larger. However, if we increase the thickness of magnets further, the flux density through the yoke becomes saturated. Then, it can pass no more flux density to the air gap. As shown in Table 2, we found that the air gap flux density could be increased to $0.810 \mathrm{~T}$ while the level of saturation of the yokes changed insignificantly. The corresponding thickness of the magnets and yokes were $2.4 \mathrm{~mm}$ and $1.8 \mathrm{~mm}$, respectively. In this case, the Halbach magnetic circuit gains an $11.3 \%$ increase in the air gap flux density compared with the conventional magnet circuit.

\section{Experimental Results}

In order to experimentally verify the effectiveness of the Halbach magnetic circuit, we fabricated a prototype and compared its performance with that of a conventional magnetic circuit equipped in a commercial HDD (3.5 inches, Samsung electronics). The magnet thickness for Halbach magnetic circuit was $2.4 \mathrm{~mm}$ from the previous

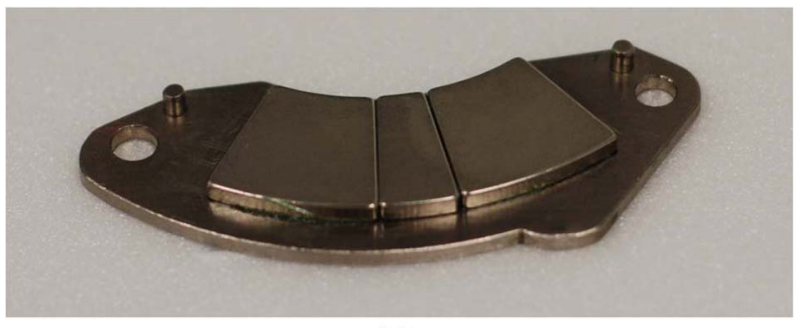

(a)

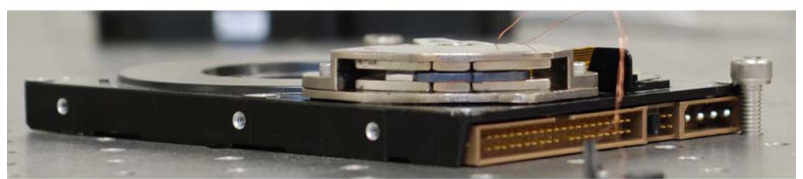

(b)

Fig. 5. Pictures of the manufactured Halbach magnetic circuit: (a) Halbach magnet array on the base yoke, (b) the installed Halbach magnetic circuit. 


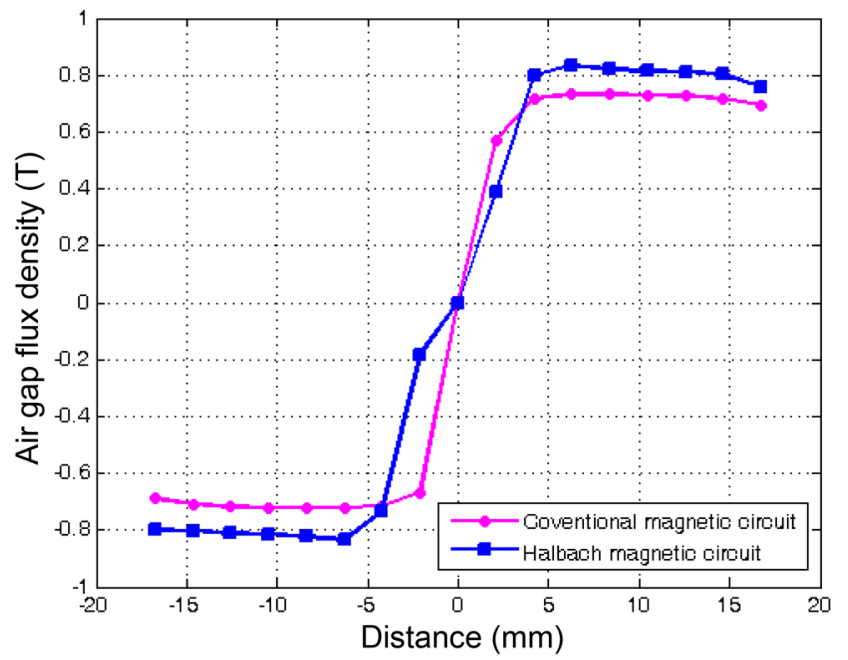

Fig. 6. Measured air gap flux density of conventional and halbach magnetic circuit.

analysis. All magnet properties were same as those used in simulations. The fabricated Halbach magnetic circuit is demonstrated in Fig. 5. In Fig. 5(a), the Halbach magnet array was assembled to the base yoke. The cover yoke, which have a same Halbach magnet array, was removed for clarity. Each magnet was glued by epoxy adhesive. Fig. 5(b) shows a side view of the Halbach magnetic circuit, which was installed in a commercial HDD instead of a conventional magnetic circuit.

The measured air gap flux density is plotted in Fig. 6 . The flux density was measured at regular spaces using gauss meter (Model 7030, F. W. BELL). The experimental measurements showed excellent agreement with the simulation results. The discrepancy was within $1 \%$ for both magnetic circuits. According to these results, the air gap flux density in the Halbach magnetic circuit was $0.816 \mathrm{~T}$, while that of the conventional magnetic circuit was 0.730 T. Therefore, there is an $11.8 \%$ increase in the air gap flux density by adopting the Halbach magnetic circuit on a rotary-type VCM. To verify the enhancement of dynamic performance, frequency responses of displacement of the VCM in response to input current were tested for both magnetic circuits: the conventional and the Halbach magnetic circuit. The displacement is measured by a laser Doppler vibrometer (OFV3001 and OFV501, Polytec) at the end-tip of the swing arm which are actuated by the VCM. A dynamic signal analyzer (35670A, Agilent) records the displacement simultaneously sweeping the input current, from $100 \mathrm{~Hz}$ to $10,000 \mathrm{~Hz}$. Fig. 7 shows the frequency response functions. Obviously, the Halbach magnetic circuit has higher magnitude than the conventional one in most ranges of frequency. Typically a

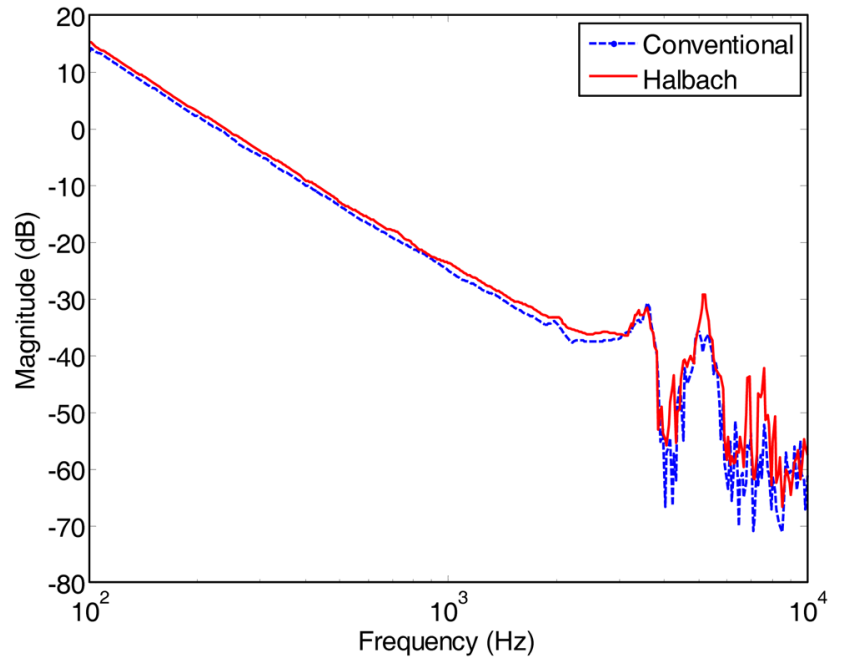

Fig. 7. Frequency response functions.

VCM is modeled as a double integrator. In this case, the corresponding magnitude is proportion to a force constant which is generated force per ampere. On an average from $100 \mathrm{~Hz}$ to $1,000 \mathrm{~Hz}$, the Halbach magnetic circuit gained $0.92 \mathrm{~dB}$ more in magnitude, which means $11 \%$ larger force constant. This result was also perfectly matched with the measured flux density.

\section{Conclusions}

In this paper, the adoption of a Halbach magnetic circuit for a rotary-type VCM was proposed. A prototype of the Halbach magnetic circuit was fabricated, and its performance was compared with the performance of a conventional magnetic circuit. We achieved an $11 \%$ increase in the dynamic force by applying the proposed Halbach magnetic circuit to a commercial HDD VCM without changing the total thickness, the air gap thickness, or the magnet surface area.

\section{Acknowledgement}

This research was supported by a Korea University Grant.

\section{References}

[1] L. P. Maguire, S. Szilagyi, and R. E. Scholten, Rev. Sci. Instrum. 75, 3077 (2004).

[2] Abdullah Al Mamun, GuoXiao Guo, and Chao Bi, Hard Disk Drive: Mechatronics and Control, CRC press, Boca Raton (2006).

[3] Haeng-Soo Lee, Young-Hoon Kim, Tae-Yeon Hwang, 
and Cheol-Soon Kim, IEEE Trans. Magn. 41, 774 (2005).

[4] Kenji Suzuki, Shingo Tsuda, Takaaki Deguchi, and Toshihiko Shimizu, Microsyst. Technol. 13, 1093 (2007).

[5] K. Halbach, Nucl. Instrum. Methods 169, 1 (1980).

[6] D. L. Trumper, W.-J. Kim, and M. E. Williams, IEEE Trans. Ind. Appl. 32, 371 (1996).

[7] W. H. Chang, J. H. Chen, and L. P. Hwang, Magn. Reson. Imaging 24, 1095 (2006).
[8] Sung-Q Lee, Kang-Ho Park, Mun-Cheal Paek, and Kwang-Yong Kang, J. Appl. Phys. 45, 1131 (2006).

[9] Jaehwa Jeong, Moon G. Lee, Jun-Hee Lee, Hyoung-Kil Yoon, and Dae-Gab Gweon, Jpn. J. Appl. Phys. 43, 1398 (2004).

[10] Moon G. Lee, Jaehwa Jeong, and Dae-Gab Gweon, Jpn. J. Appl. Phys. 42, 3394 (2003). 